\title{
Systemic biomarkers of inflammation, oxidative stress and tissue injury and repair among waterpipe, cigarette and dual tobacco smokers
}

\author{
Naushad Ahmad Khan, ${ }^{1}$ Gina Lawyer, ${ }^{1}$ Samantha McDonough, ${ }^{1}$ Qixin Wang, ${ }^{1}$ \\ Noura O Kassem, ${ }^{2}$ Flora Kas-Petrus, ${ }^{2}$ Dongxia Ye, ${ }^{1}$ Kameshwar P Singh, ${ }^{1}$ \\ Nada OF Kassem, ${ }^{2}$ Irfan Rahman (D) ${ }^{1}$
}

\begin{abstract}
'Department of Environmental Medicine, University of Rochester, Rochester, New York, USA

${ }^{2}$ Hookah Studies Division, Center for Behavioral Epidemiology and Community Health, San Diego State University, San Diego, California, USA
\end{abstract}

\section{Correspondence to}

Dr Irfan Rahman, Department of Environmental Medicine, University of Rochester, Rochester, NY 14642, USA; Irfan_Rahman@URMC.

Rochester.edu

NAK and GL contributed equally.

Received 22 January 2019 Revised 7 June 2019 Accepted 10 June 2019 Published Online First 7 September 2019

\section{ABSTRACT}

Background Waterpipe tobacco (WPT) smoking is associated with deleterious effects on cardio-pulmonary systems which may have adverse repercussions in pathophysiology and progression of chronic lung and cardiovascular diseases. We compared the biomarkers of systemic inflammation, lipid mediators, injury/repair and oxidative stress between groups of non-smokers (NS), exclusive WPT smokers (WPS), exclusive cigarette smokers (CS) and dual WPS and CS (DS).

Methods Two cohorts were recruited. Cohort I consisted of WPS $(n=12), C S(n=26)$, DS $(n=10)$ and NS $(n=25)$. Cohort II consisted of WPS $(n=33)$ and NS $(n=24)$. Plasma and urine samples were collected and analysed for various systemic biomarkers.

Results Compared with NS, plasma levels of inflammatory mediators (interleukin (IL)-6, IL-8, IL $1 \beta$ and tumor necrosis factor- $\alpha$ ) were significantly higher in WPS and CS, and were further augmented in DS. Endothelial biomarkers (intracellular adhesion molecule-1, prostaglandin E-2 and metalloproteinase-9) were significantly higher in CS. Most notably, proresolving lipid mediator (resolvin E1) and biomarkers of immunity, tissue injury, and repair were significantly lower in WPS and CS. Urinary levels of 8-isoprostane were significantly higher in all smoking groups in cohort I, while 8-isoprostane, myeloperoxidase, receptor for advanced glycation end products (RAGE), En-RAGE and matrix metalloproteinase-9 were significantly higher in all smoking groups in cohort II.

Conclusions Biomarkers of inflammation, oxidative stress, immunity, tissue injury and repair were elevated in WPS and CS groups. Furthermore, concurrent use of WPT and cigarettes is more harmful than cigarette or WPT smoking alone. These data may help inform the public and policy-makers about the dangers of WPT smoking and dual use of tobacco products.

\section{INTRODUCTION}

Waterpipe tobacco (WPT) smoking, also known as hookah, shisha and narghile, is gaining popularity among youth in western countries including the USA. ${ }^{1}$ It is commonly perceived as less harmful than cigarette smoking. However, studies have shown that WPT smoking manifests similar acute and chronic health effects as cigarette smoking. ${ }^{2} 3$ Like cigarette smoking, WPT smoking can also increase the risk of cardiovascular disease, chronic bronchitis and cancer. ${ }^{34}$
WPT smoke contains similar concentrations of toxic chemicals and carcinogens (eg, polycyclic aromatic hydrocarbons and aldehydes) as cigarette smoke. ${ }^{5}$ Moreover, WPT and cigarette smoking have been shown to be risk factors for periodontal and cardiopulmonary diseases. ${ }^{78}$ Similarly, other studies have shown that daily chronic use of WPT can lead to chronic obstructive pulmonary disease (COPD) and significant reduction in forced expiratory volume- 1 and forced vital capacity. ${ }^{9}$

While biomarkers of systemic inflammation and oxidative stress associated with cigarette smoking are well characterised, few studies have examined the association of such biomarkers with WPT use. Profiling blood and urine biomarkers from CS and WPT smokers (WPS) can help us understand the differential toxicity of cigarette and WPT smoking in the pathogenesis of various cardiopulmonary diseases. WPT smoking may be associated with systemic inflammation and oxidative stress in the lungs which can be persistent and can lead to cardiovascular and pulmonary diseases. ${ }^{1011} \mathrm{~A}$ recent epidemiological survey showed that dual smokers (DS) have a higher frequency of WPT smoking than WPS alone. ${ }^{12}$ Given emerging rates of concurrent use of WPT and cigarettes among young adults, ${ }^{12} 13$ it is also important to understand whether dual use poses a greater threat to health than exclusive WPT use.

In this study, we measured and compared the levels of several biomarkers of toxicity in plasma and urine samples among an exclusive group of waterpipe smokers (WPS), cigarette smokers (CS), dual smokers (DS) and non-smokers (NS). We hypothesised that, compared with exclusive WPT and cigarette use, dual use would enhance and modulate systemic inflammation, oxidative stress and repair processes in the lungs.

\section{SUBJECTS AND METHODS Participant recruitment, screening and consent: cohort I and cohort II}

We used samples and data from two different populations in the USA. The first cohort study (cohort I) was a cross-sectional study conducted at the University of Rochester Medical Center in Rochester, New York. The second cohort study (cohort II) was a community-based study conducted at the Center for Behavioral Epidemiology and Community Health, Hookah Studies Division, San Diego State University in San Diego, California. 
Table 1 Baseline characteristics of study participants according to smoking status

\begin{tabular}{|c|c|c|c|c|c|}
\hline Cohort I & Non-smokers $(n=25)$ & Cigarette smokers $(n=26)$ & Waterpipe smokers $(n=12)$ & \multicolumn{2}{|c|}{ Dual smokers $(n=10)$} \\
\hline Age (years) & $36.16 \pm 12.52$ & $46.73 \pm 9.96$ & $32.84 \pm 14.04$ & \multicolumn{2}{|l|}{$39.5 \pm 12.49$} \\
\hline Male & $37.53 \pm 10.95$ & $47 \pm 9.96$ & $35 \pm 16.26$ & \multicolumn{2}{|l|}{$37.5 \pm 14.84$} \\
\hline Female & $34.66 \pm 14.37$ & $46.46 \pm 10.95$ & $28 \pm 6.16$ & \multicolumn{2}{|l|}{$42.5 \pm 9.0$} \\
\hline \multicolumn{6}{|l|}{ Gender } \\
\hline Male & $13(52 \%)$ & $13(50 \%)$ & $9(69.24 \%)$ & \multicolumn{2}{|l|}{$6(60 \%)$} \\
\hline Female & $12(48 \%)$ & $13(50 \%)$ & $4(30.76 \%)$ & \multicolumn{2}{|l|}{$4(40 \%)$} \\
\hline \multicolumn{4}{|l|}{ Smoking status } & CS & WPS \\
\hline Smoking frequency* & $\mathrm{N} / \mathrm{A}$ & $12.89 \pm 6.39$ & $1.45 \pm 0.93$ & $9.13 \pm 6.06$ & $2.09 \pm 1.30$ \\
\hline Duration of smoking (years) $\dagger$ & N/A & $20.03 \pm 8.55$ & $2.72 \pm 1.84$ & $14.00 \pm 12.89$ & $4.69 \pm 3.72$ \\
\hline Duration of smoking per session (minutes) $\ddagger$ & $\mathrm{N} / \mathrm{A}$ & $\mathrm{N} / \mathrm{A}$ & $15.00 \pm 15.17$ & $\mathrm{~N} / \mathrm{A}$ & $16.40 \pm 16.55$ \\
\hline Cohort II & \multicolumn{2}{|l|}{ Non-smokers $(n=24)$} & \multicolumn{3}{|l|}{ Waterpipe smokers $(n=33)$} \\
\hline Age (years) & \multicolumn{2}{|l|}{$25.9 \pm 4.45$} & \multicolumn{3}{|l|}{$25.0 \pm 3.10$} \\
\hline Male & \multicolumn{2}{|l|}{$26.0 \pm 4.90$} & \multicolumn{3}{|l|}{$26.0 \pm 2.83$} \\
\hline Female & \multicolumn{2}{|l|}{$25.8 \pm 4.02$} & \multicolumn{3}{|l|}{$24.1 \pm 3.07$} \\
\hline \multicolumn{6}{|l|}{ Gender } \\
\hline Male & \multicolumn{2}{|l|}{$11(45.8 \%)$} & \multicolumn{3}{|l|}{$16(48.5 \%)$} \\
\hline Female & \multicolumn{2}{|l|}{$13(54.2 \%)$} & \multicolumn{3}{|l|}{$17(51.5 \%)$} \\
\hline \multicolumn{6}{|l|}{ Smoking Status } \\
\hline Smoking Frequency* & \multicolumn{2}{|l|}{$\mathrm{N} / \mathrm{A}$} & \multicolumn{3}{|l|}{$1.3 \pm 0.51$} \\
\hline Duration of smoking (years) $\dagger$ & \multicolumn{2}{|l|}{$\mathrm{N} / \mathrm{A}$} & \multicolumn{3}{|l|}{$4.20 \pm 3.32$} \\
\hline
\end{tabular}

Data are expressed as mean \pm SD or as the number of subjects (\%).

All biological samples collected were not used for all biomarkers analysis. Number of subjects used for biomarkers analyses are given in parenthesis in Tables $2 \mathrm{~A}$ and $2 \mathrm{~B}$.

${ }^{*}$ Average number of times subjects smoked cigarettes, waterpipe tobacco or both per day.

tMean of numbers of years subjects have been smoking cigarettes, waterpipe tobacco or both.

$\ddagger$ Average duration of each smoking session.

CS, cigarette smokers; N/A, not applicable; WPS, waterpipe tobacco smokers.

For cohort I, study participants were recruited/enrolled at the General Clinical Research Center of the University of Rochester via various local newspaper and magazine advertisements, word of mouth, hookah lounges and flyers posted in and around the university campus. Based on a self-reported questionnaire containing information about demographic variables, smoking history and smoking behaviour, the study participants were categorised into four groups: healthy NS $(n=25)$, CS $(n=26)$, WPS $(n=12)$ and DS $(n=10)$. The analysed samples and their respective ' $n$ ' are given in figure legends and tables from the above cohort. Samples were analysed to accommodate the assay of all the mediators in relatively equal numbers in all the groups. Table 1 shows the demographic information for all participants; those in cohort I had a demographic profile similar to those in cohort II.

All the potential study subjects were screened for eligibility via telephone using the following eligibility criteria: (1) age 21-65 years; (2) NS without the use of any tobacco products (healthy NS); (3) smokers (CS, WPS and DS) currently smoking $\geq 10$ cigarettes and/or 1-2 waterpipe sessions per day for $\geq$ past 6 months; (4) none of the subjects (NS, CS, WPS and DS) having history of chronic illnesses, such as lung and heart diseases, diabetes and cancer; (5) none of the subjects having any current respiratory/ pulmonary infections (viral-flu, pneumonia) or on any prescribed anti-inflammatories and/or corticosteroids and (6) female subjects not currently pregnant or breast feeding. Written informed consent was obtained from all study subjects prior to participation in the study in both the cohorts (Cohorts I and II).

Cohort II included a convenience sample of exclusive WPS $(n=33)$ and NS $(n=24)$ recruited between December 2017 and September 2018, from San Diego County, California, USA. Subjects were recruited via flyers posted on bulletin boards at community colleges, universities and cafes; and electronic flyers posted on social media. WPS, 21 years or older, who smoke WPT exclusively, smoke at least one WPT head per month, smoke at least one WPT head per smoking session and smoke WPT at home were enrolled in the study. Participants with a history of chronic health problems (eg, asthma, COPD, hypertension); regular use of prescription medication (other than vitamins or birth control); or pregnancy were excluded from the study. All the study participants provided written informed consent. Non-smoking status was based on self-report and exhaled carbon monoxide cut-off levels (cut-off-point: mean $\pm \mathrm{SD}=3.61 \pm 2.15 \mathrm{ppm}$ ). ${ }^{13}$ Table 1 shows the demographic information for the entire sample; participants in cohort II had a demographic profile similar to those in cohort I.

\section{Study design}

Cohort I: This study is a cross-sectional analysis of biomarkers of tobacco product exposure. Participants were recruited as part of an ongoing study at the University of Rochester to evaluate the blood and urine biomarkers of inflammation, oxidative stress and lipid mediators. Whole venous blood (20-25 mL) was collected from participants in vacutainer tubes containing EDTA, and plasma was processed and separated by centrifugation for 10-15 min at 2000 $\mathrm{g}$ within $60 \mathrm{~min}$ of collection. The supernatants were aspirated and aliquoted into several tubes for multiple assessments, and stored immediately at $-80^{\circ} \mathrm{C}$ until analysed. Study participants also provided urine samples $(20-25 \mathrm{~mL})$ which were aliquoted and stored in a freezer $\left(-20^{\circ} \mathrm{C}\right)$, until analysed.

Cohort II: A detailed descriptions of data collection of urine samples, demographics and WPT use behaviours has been previously published. ${ }^{1415}$ Briefly, first void urine samples were collected the morning following the day smokers smoked a single session of WPT at home. 

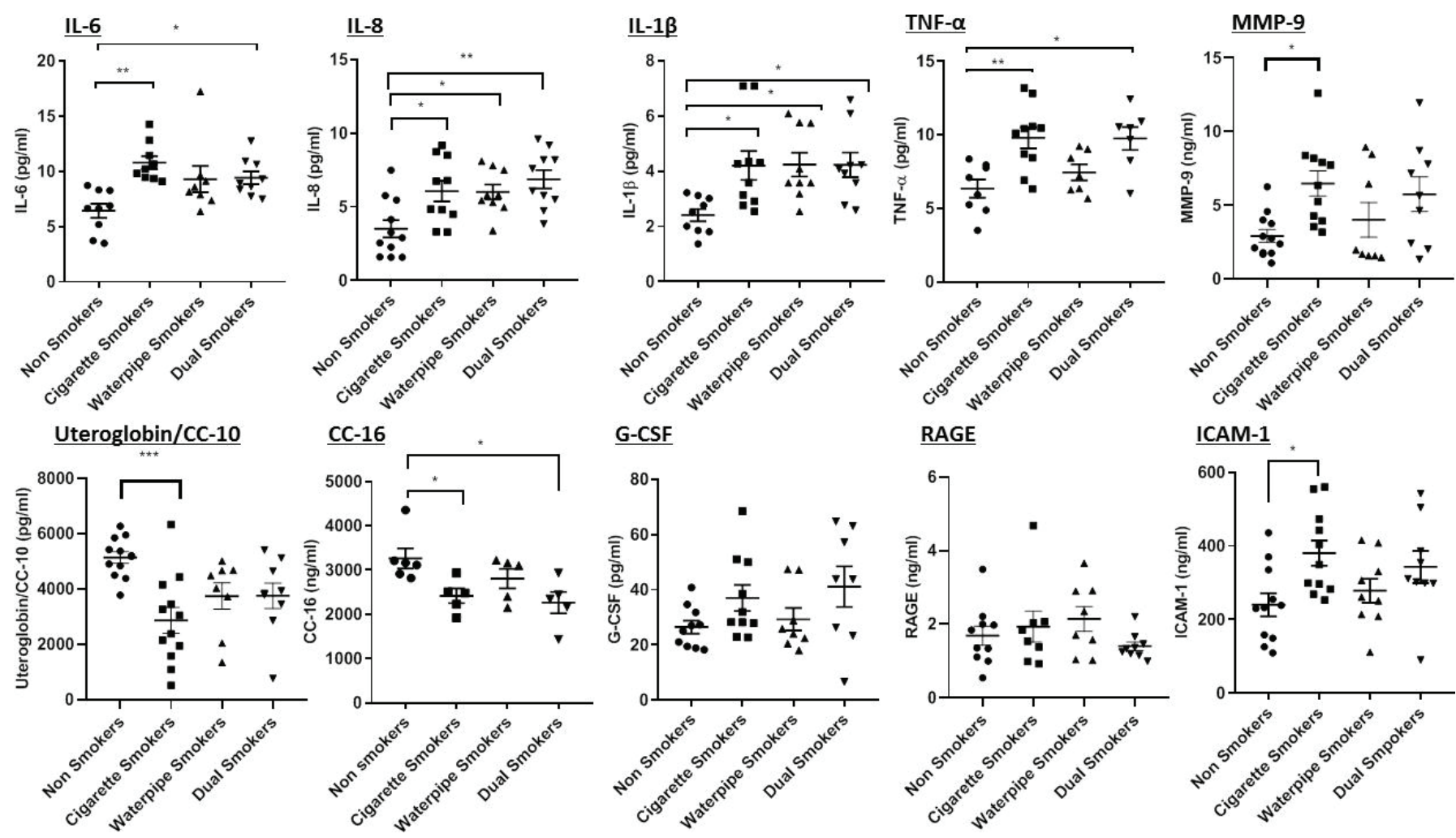

Figure 1 Biomarkers of systemic inflammation and oxidative stress measured in plasma. Quantification of systemic inflammatory and endothelial activation biomarkers in plasma of non-smokers, cigarette, waterpipe tobacco and dual smokers. Data are presented as mean \pm SEM ( $n=5-11)$. Statistical significance was determined by one-way analysis of variance using multiple comparison with Tukey's post hoc analysis for comparison between the different groups. ${ }^{*} \mathrm{P}<0.05,{ }^{*} \mathrm{P}<0.01,{ }^{* *} \mathrm{P}<0.001$. G-CSF, granulocyte-colony stimulating factor; IL, interleukin; MMP, matrix metalloproteinase-9; RAGE, receptor for advanced glycation end products; ICAM-1, intracellular adhesion molecule-1; TNF- $\alpha$, tumor necrosis factor- $\alpha$; CC-16, Club cell protein-16.

\section{Measures}

Demographics and smoking assessment

Study participants provided information regarding demographic characteristics, such as age, sex, gender and ethnicity. Detailed information about the use of WPT and cigarettes, smoking frequency and duration of tobacco product use, allowed us to categorise participants into four groups of smokers.

\section{Measurements of plasma cotinine and urinary 4-(methylnitrosamino)-1-(3-pyridyl)-1-butanol and its glucuronides}

Plasma cotinine was measured to determine smoking status and tobacco smoke exposure using Salimetrics' (Carlsbad, CA, USA) high sensitivity quantitative enzyme immunoassay kit according to manufacturer's instructions. Urinary levels of 4-(methylnitrosamin o)-1-(3-pyridyl)-1-butanol and its glucuronides (NNAL) measured using Waters Xevo TQ-XS Tandem Mass Spectrometer with ACQUITY UPLC I-Class Chromatography System (UPLC-MS/ MS) by the NicoTAR laboratory at Roswell Park Cancer Institute, Buffalo, New York.

\section{Measurement of biomarkers by multiplex panel assay}

Plasma levels of cytokines and chemokines were profiled using human XL18-plex magnetic Luminex immunoassay kit. Biomarkers of immunity, tissue injury and repair were measured in plasma and urine samples by using human XL 9-plex magnetic Luminex immunoassay kit (catalogue nos. FCSTM18-18/17 and LXSAHM-09 respectively, R\&D systems, Minnesota, USA), based on a pre-optimised protocol as per manufacturer's instructions. Out of range values were designated as the lowest detectable value ' 0 '. The plasma concentrations of interleukin (IL)-6, IL-8, IL-10, IL-13, IL-1 $\beta$, IL-1 $\alpha$, IL-33 and interferon- $\gamma$, Eotaxin, MIP-1 $\alpha$, MIP-1 $\beta$, granulocyte-colony stimulating factor (G-CSF), GM-CSF, MCP-1, Gro alpha/CXCL-1, Gro beta/CXCL-2 and TNF- $\alpha$, were measured by Luminex multiplex assay. Additionally, S100A8, S100A9, plasminogen activator inhibitor-1 (Serpine1/ PAI-1), matrix metalloproteinase-9 (MMP-9), myeloperoxidase (MPO), galectin -3 , receptor for advanced glycation end products (RAGE), Uteroglobin/CC-10 and ligand for the RAGE (En-RAGE) were measured in urine and plasma samples by Luminex multiplex assay. Samples were assayed in duplicates and values were reported as the average of the duplicated samples.

\section{Measurement of plasma and urine biomarkers by ELISA}

Plasma levels of c-reactive protein (CRP) were measured using commercially available ELISA kits (Sigma-Aldrich, St. Louis, Missouri, USA). The levels of 8-isoprostanes, prostaglandin $\mathrm{E}_{2}$ ( $\left.\mathrm{PGE}_{2}\right)$, thromboxane $\mathrm{B}_{2}$ (Cayman, Ann Arbour, MI), resolvin E1 (MyBiosource, San Diego, USA), fibrinogen (Abcam, Cambridge, Massachusetts, USA), human CC10/CC16 (MyBiosource, San Diego, USA), (human synonym name for mouse CC16 is CC10), pentraxin 3 (Sigma, St. Louis, USA), Intracellular Adhesion Molecule-1 (RD Systems, Minneapolis, USA), desmosine (MyBiosource, San Diego, USA), NT-proBNP (RD Systems, Minneapolis, USA) and malondialdehyde (MDA) (Cell Biolabs, San Diego, USA) were measured quantitatively by commercially available ELISA kits as per manufacturer's instructions. 
A Thromboxane $\underline{B}_{2}$

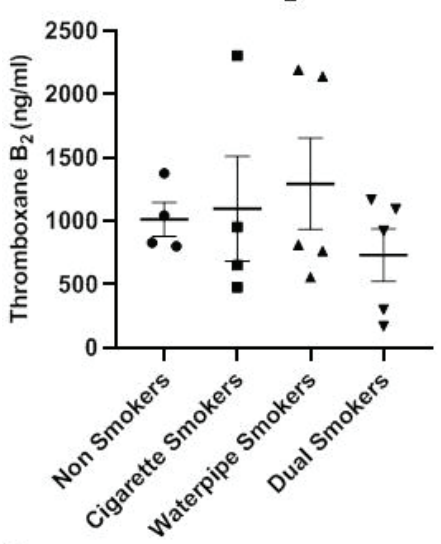

B

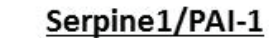

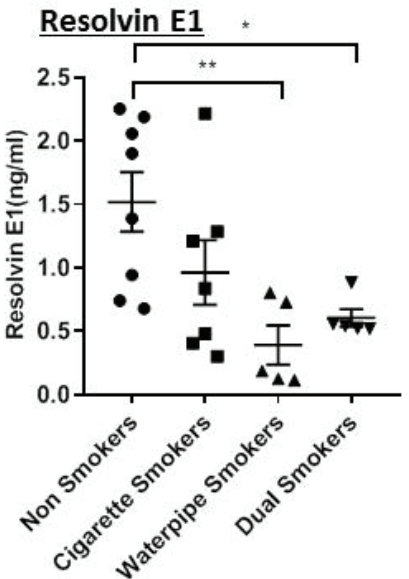

$\underline{\text { CRP }}$

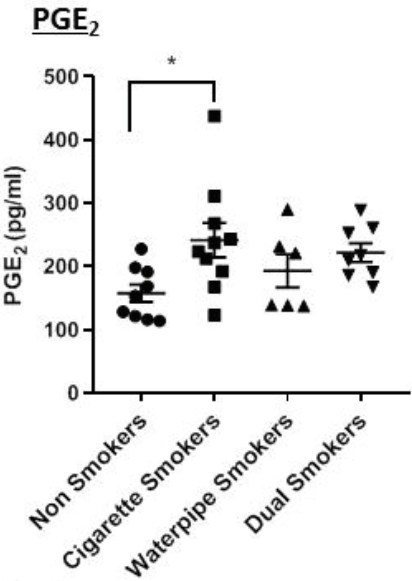

Fibrinogen

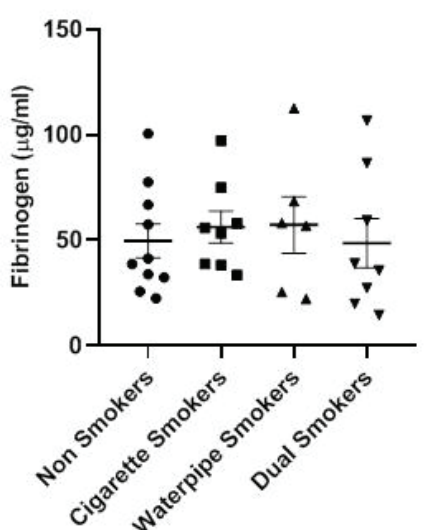

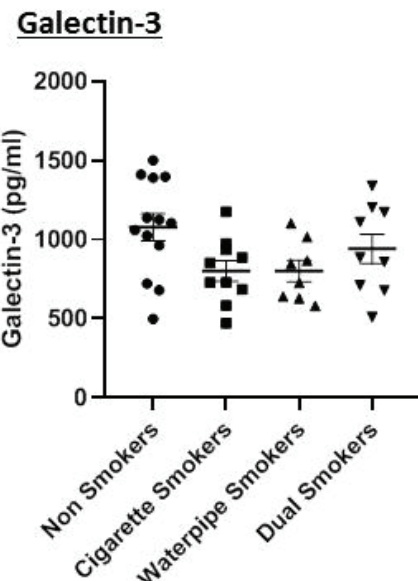

Figure 2 Plasma levels lipid mediators and biomarkers of immunity, tissue and repair and stress mediators. Plasma levels of (A) lipid mediators and (B) biomarkers of immunity, tissue injury/repair and stress mediators were determined in non-smokers, cigarette smokers, waterpipe tobacco smokers and dual smokers. Data are presented as mean \pm SEM $(n=4-10)$. Statistical significance was determined by one-way analysis of variance using multiple comparison with Tukey's post hoc analysis for comparison between the different groups. ${ }^{*} \mathrm{P}<0.05,{ }^{*} \mathrm{P}<0.01$. PAl-1, plasminogen activator inhibitor-1; $\mathrm{PGE}$, prostaglandin $\mathrm{E}_{2} ; \mathrm{CRP}$, c-reactive protein.

\section{Data and statistical analysis}

Statistical analyses were performed using GraphPad Prism Software V.7.0. Categorical variables are presented as a number (percentage), and continuous variables are presented as the mean \pm SEM unless otherwise indicated. Statistical significance of urine samples biomarkers from cohort II was determined by Student's t-test. Statistical significance of cohort I plasma and urine samples biomarkers was calculated using one-way analysis of variance followed by Tukey's post hoc test for multiple comparisons. $\mathrm{P}$ value of $<0.05$ was accepted as statistically significant.

\section{RESULTS}

\section{Demographic and clinical findings}

Table 1 summarises the baseline clinical, demographic and functional characteristics of the study participants. A total of 73 study participants were recruited in the study (cohort I). Mean age of the participants in the CS group was comparatively higher compared with NS, WPS and DS groups. Female participants were comparatively younger than male participants in all the groups except DS group. Daily smoking frequency (sessions), mean duration of smoking and average duration of each smoking session in each group are given in table 1. A majority of the participants were white followed by African Americans, Asians, Hispanics and Pacific Islanders. Plasma cotinine levels were found to be significantly higher in CS $(83.76 \pm 26.36$ $\mathrm{ng} / \mathrm{mL}, \mathrm{p}<0.001)$, and DS $(150.29 \pm 29.16 \mathrm{ng} / \mathrm{mL}, \mathrm{p}<0.001)$, but non-significant in WPS $(11.95 \pm 5.69 \mathrm{ng} / \mathrm{mL})$ compared with NS $(0.44 \pm 0.12 \mathrm{ng} / \mathrm{mL})(\mathrm{n}=8-18$ for all groups). Urinary levels of NNAL were increasingly higher in CS $(32.53 \pm 8.45 \mathrm{pg} / \mathrm{mg}$ creatinine; $\mathrm{n}=12, \mathrm{p}<0.01)$, WPS $(34.85 \pm 12.17 \mathrm{pg} / \mathrm{mg}$ creatinine; $\mathrm{n}=8$, $\mathrm{p}<0.01)$ and DS $(45.92 \pm 14.86 \mathrm{pg} / \mathrm{mg}$ creatinine; $\mathrm{n}=10 ; \mathrm{p}<0.01)$ compared with NS $(0.00 \mathrm{pg} / \mathrm{mg}$ creatinine; $\mathrm{n}=12)$, suggesting an active smoking of tobacco products.

Biomarkers of systemic inflammation, oxidative stress, lipid mediators and immunity, tissue injury/repair and stress mediators in plasma

To determine whether the systemic inflammatory response was different among WPS, CS, DS and NS, plasma levels of IL-6, IL-8, IL-1 $\beta$, TNF $\alpha$, MMP-9, CC-10, CC-16, G-CSF and RAGE were measured. Levels of IL-6, IL-8, TNF $\alpha$ and IL-1 $\beta$ in plasma were significantly higher in WPS, CS and DS compared with NS (figure 1). Clara/Club cell proteins, such as CC-10 and/or CC-16 were significantly lower in CS with a trend of lower levels in other smoking groups compared with NS (figure 1). Soluble receptor for advanced glycation product (sRAGE), a marker of tissue injury and inflammation and G-CSF had no significant changes in all smoking groups compared with NS. Of all the studied biomarkers of systemic inflammation, IL-1 $\beta$ and IL- 6 values had significant increases in all smoking groups compared with NS (figure 1). Other inflammatory mediators, 
such as IL-13, CXCL-1, CXCL-2, MIP1 $\alpha$, MIP-1 $\beta$, eotaxin, GM-CSF, MCP-1, S100A8 and S100A9 showed slight but insignificant elevation, whereas IL-10 was reduced in CS, WPS and DS versus NS (table $2 \mathrm{~A}$ ). IL- $1 \alpha$, IL-33 and IF- $\gamma$ showed variable and insignificant results (data not shown).

Biomarkers of endothelial dysfunction and oxidative stress, such as ICAM-1, MDA and MPO were measured in plasma. ICAM-1 levels were significantly increased in CS compared with NS (figure 1), but without any significant changes seen for MPO levels in both CS and DS as compared with NS (table 2A). There were no significant changes in levels of MDA in plasma samples of all smoking groups versus NS.

To determine the possible role of lipid mediators as biomarkers of exposure to tobacco products, we measured plasma levels of thromboxane $\mathrm{B}_{2}$, resolvin $\mathrm{E}_{1}$ and PGE-2 in plasma samples of NS, CS, WPS and DS. Plasma levels of resolvin E1 were significantly decreased in WPS and DS compared with NS (figure 2A). Plasma levels of $\mathrm{PGE}_{2}$ were elevated significantly in CS compared with NS. Thromboxane $\mathrm{B}_{2}$ showed no significant changes between groups.

Biomarkers of immunity, tissue injury /repair and stress mediators, such as Serpine1/PAI-1, CRP, fibrinogen, galectin-3, MMP-9, S100A8 and S100A9 were also measured in plasma. There was a trend toward higher levels of damage associated molecular patterns, such as S100A8 in the plasma of all smoking groups compared with NS, but the difference was not statistically significant (table 2A). The markers of atherosclerotic lesion in response to inflammatory stimuli (Pentraxin-3), stress (CRP), serpine-1/PAI-1, heart function (NT-proBNP) and elastin breakdown (desmosine) did not show significant changes in any of the smokers (CS, WPT and DS) groups (table 2A).
Biomarkers of inflammatory mediator and coronary heart disease, oxidative stress and tissue injury and repair in urine We extended our assessment of oxidative stress markers (8-isoprostanes and MPO) and other mediators (galectin-3, RAGE, En-RAGE, MMP-9, S100A8, S100A9, Uteroglobin/CC-10 and Serpine1/PAI-1) in urine samples of both the cohorts to include a wider array of biospecimens that can be used for biomarker analysis. Levels of urinary 8-isoprostanes were significantly higher in all smoking groups with even higher levels in DS compared with NS in cohorts I (figure 3A). Urinary levels of 8-isoprostanes, MPO and RAGE were significantly higher in WPS compared with NS in cohort II (figure 3B). The level of Serpine1/PAI-1 was higher in DS only versus NS (table 2B). S100A9 was shown to be significantly lower in CS and WPS, but not DS compared with NS (table 2B). There was no significant change observed in MPO, galectin-3, RAGE, En-RAGE and MMP-9 urinary levels in cohort I in all groups versus NS (figure 3A). However, the urinary levels of En-RAGE and MMP-9 levels were elevated significantly in WPS in comparison to NS in cohort II (figure 3B). While the levels of S100A9 and Serpine1 were significantly higher compared with NS in cohort I, there was no significance seen in those biomarkers for cohort II. Other mediators, such as S100A8 and Uteroglobin/ CC-10 did not show any significant changes in any of the smoking groups compared with NS in both cohorts (table 2B).

\section{DISCUSSION}

The current study is the first to determine the differential effects of various forms of tobacco smoking, such as WPT, cigarette and DS on levels of biomarkers of inflammation, oxidative stress and immunity, and tissue injury and repair in human plasma and urine.

A
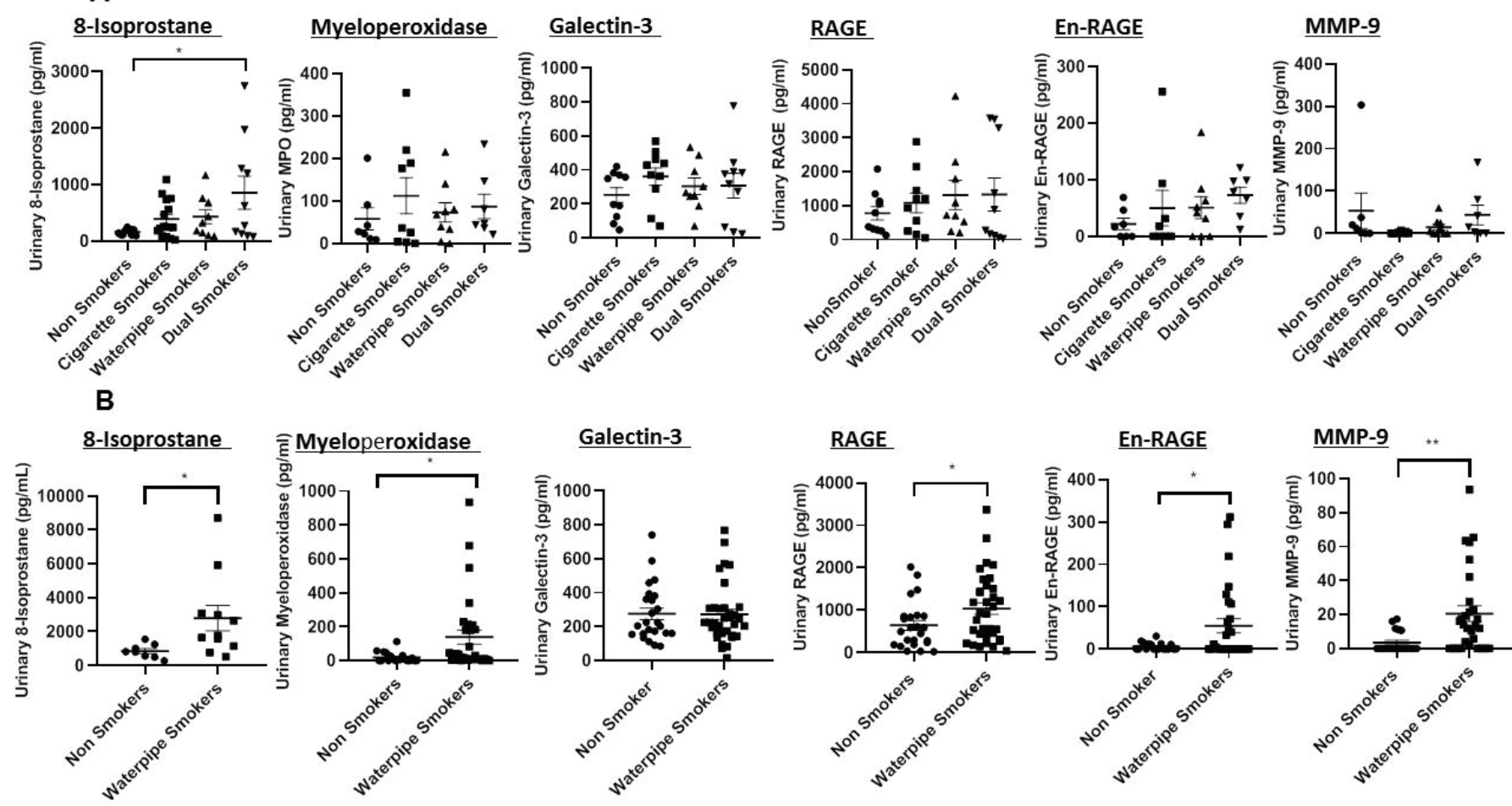

Galectin-3
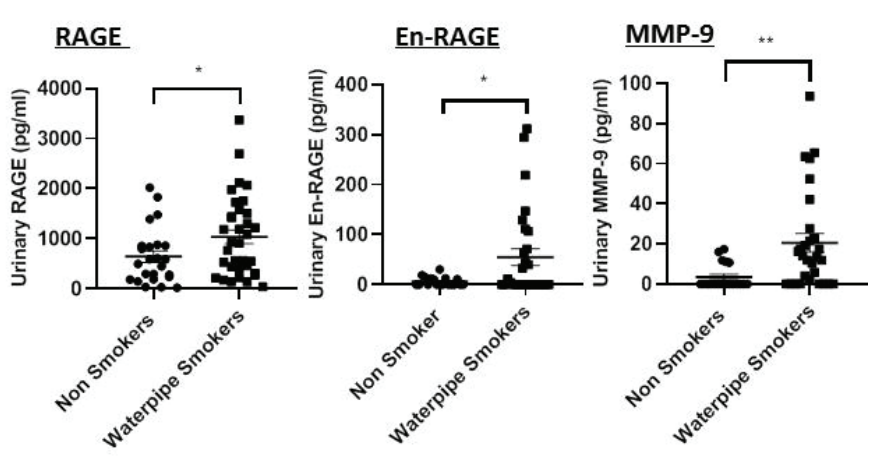

Figure 3 Biomarkers of oxidative stress and inflammation measured in urine samples of both cohorts. Urinary levels of 8-isoprostane, MPO, galectin-3, RAGE, En-RAGE and MMP-9 were determined in non-smokers, cigarette smokers, waterpipe tobacco smokers and dual smokers from cohort I (A) and between non-smokers and waterpipe smokers of cohort II (B). Data are presented as mean \pm SEM ( $n=7-33)$. Statistical significance was determined by one-way analysis of variance using multiple comparison with Tukey's post hoc analysis for comparison between the different groups for cohort I. For cohort II, statistical significance was determined by unpaired t-test. ${ }^{*} \mathrm{P}<0.05$, ${ }^{*} \mathrm{P}<0.01$. MMP-9, matrix metalloproteinase- 9 ; MPO, myeloperoxidase; RAGE, receptor for advanced glycation end products. 
Table 2A Plasma biomarkers of tobacco smoke exposure among non-smokers and cigarette, waterpipe and dual smokers

\begin{tabular}{|c|c|c|c|c|}
\hline Biomarkers & Non-smokers & Cigarette smokers & Waterpipe smokers & Dual smokers \\
\hline \multicolumn{5}{|l|}{ Inflammatory mediators } \\
\hline MIP-1 $\alpha(p g / m L, n=8-10)$ & $8.91 \pm 0.75$ & $12.97 \pm 1.56$ & $11.93 \pm 2.60$ & $14.20 \pm 1.29$ \\
\hline MIP-1 $\beta(p g / m L, n=8-10)$ & $269.20 \pm 5.55$ & $282.40 \pm 15.48$ & $274.70 \pm 27.05$ & $283.20 \pm 11.58$ \\
\hline MCP-1 (pg/mL, $\mathrm{n}=8-10)$ & $82.85 \pm 6.75$ & $98.38 \pm 8.22$ & $91.49 \pm 9.12$ & $94.95 \pm 7.79$ \\
\hline Eotaxin $(p g / m L, n=8-10)$ & $87.22 \pm 12.55$ & $122.80 \pm 15.27$ & $109.10 \pm 11.20$ & $115.70 \pm 5.33$ \\
\hline GM-CSF (pg/mL, n=8-9) & $25.77 \pm 3.10$ & $30.18 \pm 3.61$ & $26.27 \pm 3.46$ & $35.56 \pm 1.69$ \\
\hline S100A9 (pg/mL, n=8-12) & $878.70 \pm 118.50$ & $1736.00 \pm 474.50$ & $1158.00 \pm 422.20$ & $673.60 \pm 153.30$ \\
\hline En-RAGE (pg/mL, n=8-13) & $9715.00 \pm 105.5$ & $7981.00 \pm 625.8$ & $9053.00 \pm 368.1$ & $8650.00 \pm 437.9$ \\
\hline Pentraxin $3(\mathrm{ng} / \mathrm{mL}, \mathrm{n}=6-9)$ & $0.65 \pm 0.08$ & $1.02 \pm 0.24$ & $0.72 \pm 0.19$ & $0.65 \pm 0.15$ \\
\hline Interleukin-10 (pg/mL, n=7-9) & $34.77 \pm 4.91$ & $19.87 \pm 2.57$ & $30.35 \pm 10.37$ & $33.74 \pm 9.73$ \\
\hline CXCL-1 (pg/mL, n=8-10) & $252.60 \pm 37.19$ & $337.30 \pm 37.63$ & $379.90 \pm 58.09$ & $410.80 \pm 32.03$ \\
\hline Desmosine $(p g / m L, n=8-11)$ & $510.70 \pm 52.55$ & $403.50 \pm 40.86$ & $453.60 \pm 65.00$ & $452.50 \pm 25.01$ \\
\hline \multicolumn{5}{|l|}{ Oxidative stress } \\
\hline Malondialdehyde (pmol/mL, $\mathrm{n}=6-10)$ & $35.57 \pm 2.09$ & $34.90 \pm 4.83$ & $32.62 \pm 3.35$ & $51.90 \pm 8.15$ \\
\hline Myeloperoxidase (pg/mL, $\mathrm{n}=8-12)$ & $9327.00 \pm 934.50$ & $12198.00 \pm 2401.00$ & $6325.00 \pm 933.10$ & $7012.00 \pm 779.10$ \\
\hline
\end{tabular}

Data are presented as mean \pm SEM. Statistical significance was determined by one-way analysis of variance using multiple comparison with Tukey's post hoc analysis for comparison between the groups.

CXCL-1, chemokine (C-X-C motif) ligand 1 and CXC-2 (CXC motif) ligand 2; GM-CSF, granulocyte-macrophage colony-stimulating factor; MCP-1, monocyte chemoattractant protein-1; MIP-1 $\alpha$, macrophage inflammatory protein -1 alpha; MIP-1 $\beta$, macrophage inflammatory protein-1 beta; MMP-9, matrix metallopeptidase-9; $n$, number of samples; NT-proBNP, N-terminal pro-brain natriuretic peptide; S100A8, S100 calcium-binding protein A8; S100A9, S100 calcium-binding protein A9.

Table 2B Urine biomarkers of tobacco smoke exposure among non-smokers and cigarettes, waterpipe and dual smokers from cohort I and nonsmokers and waterpipe tobacco smokers from cohort II

\begin{tabular}{|c|c|c|c|c|}
\hline Biomarkers & Non-smokers & Cigarette smokers & Waterpipe smokers & Dual smokers \\
\hline \multicolumn{5}{|l|}{ Cohort I urine samples } \\
\hline \multicolumn{5}{|l|}{ Inflammatory mediators } \\
\hline S100A8 (pg/mL, n=8-10) & $392.70 \pm 131.20$ & $125.00 \pm 34.79$ & $111.90 \pm 49.11$ & $133.00 \pm 25.30$ \\
\hline S100A9 (pg/mL, n=8-10) & $385.00 \pm 101.80$ & $80.06 \pm 32.55^{*}$ & $44.96 \pm 21.51^{*}$ & $249.80 \pm 93.39$ \\
\hline Uteroglobin/CC-10 (pg/mL, n=9-10) & $1519.00 \pm 545.90$ & $1744.00 \pm 537.00$ & $3721.00 \pm 1028.00$ & $2757.00 \pm 864.00$ \\
\hline \multicolumn{5}{|l|}{ Tissue injury and repair } \\
\hline Serpine1/PAI-1 (pg/mL, n=9-10) & $1.96 \pm 0.57$ & $3.55 \pm 0.38$ & $3.81 \pm 0.59$ & $5.35 \pm 1.09^{*}$ \\
\hline Cohort II urine samples & & NA & & NA \\
\hline \multicolumn{5}{|l|}{ Inflammatory ediators } \\
\hline S100A8 (pg/mL, n=22-26) & $307.50 \pm 74.64$ & & $233.40 \pm 81.47$ & \\
\hline $\mathrm{S} 100 \mathrm{~A} 9(\mathrm{pg} / \mathrm{mL}, \mathrm{n}=20-29)$ & $765.50 \pm 261.60$ & & $400.70 \pm 105.60$ & \\
\hline Uteroglobin/CC-10 (pg/mL, $\mathrm{n}=21-29)$ & $744.13 \pm 176.90$ & & $1053.00 \pm 190.70$ & \\
\hline \multicolumn{5}{|l|}{ Tissue injury and repair } \\
\hline Serpine1/PAl-1 (pg/mL, $n=24-33)$ & $1.45 \pm 0.89$ & & $1.24 \pm 0.41$ & \\
\hline
\end{tabular}

Data are presented as mean \pm SEM. Statistical significance was determined by one-way analysis of variance using multiple comparison with Tukey's post hoc analysis for comparison between the groups in cohort I. Statistical significance of urine biomarkers between the two groups was determined by Student's t-test in cohort II. * P $<0.05$. ${ }^{*} \mathrm{P}<0.05$

n, number of samples; NA, not applicable;PAl-1, plasminogen activator inhibitor-1; S100A8, S100 calcium-binding protein A8; S100A9, S100 calcium-binding protein A9.

In this cross-sectional study, we assessed more than thirty putative blood biomarkers belonging to different panels to characterise them as signature biomarkers of WPS, CS and DS. The analytes we have chosen have been shown to be associated with systemic inflammation, oxidative stress and immunity, and tissue injury and repair in the pathogenesis of COPD and cardiovascular diseases. Systemic inflammation and oxidative stress have been shown to be important events in respiratory and cardiovascular disorders. ${ }^{16} 17$
Plasma levels of inflammatory biomarkers IL-6, IL-8, TNF- $\alpha$ and IL-1 $\beta$ were significantly higher in CS, WPS and DS compared with NS. Previous studies have shown that levels of these inflammatory markers are significantly elevated in plasma samples of patients with COPD and cardiovascular complications compared with controls. ${ }^{18-21}$ These cytokines and mediators play an important role in inflammatory pathways that can lead to tissue damage in the lungs. ${ }^{22}$ Our findings are consistent with previous clinical 
studies that have shown elevated levels of IL- 6 and IL1 $\beta$ in WPT and e-cigarette users compared with NS in periodontal disease. ${ }^{24-26}$ The results on inflammatory biomarkers suggest that concurrent use of WPT and cigarette smoking may be a potential risk factor for developing chronic respiratory and cardiovascular disease.

We hypothesised that WPT and dual smoking can further aggravate oxidative modification of important biologic molecules such as 8 -isoprostanes, MDA and MPO. Although there was a trend towards increased levels of MPO in CS, there were no significant differences seen in other groups, suggesting that smoking causes oxidative modification of biological components in human plasma. Also, we were able to show that 8-isoprostane (in both cohorts), and MPO (cohort II) levels were increased in urine samples, suggesting that these mediators can be used as a biomarker of oxidative stress and inflammation for WPT exposure. A recent meta-analysis comparing 18 studies has shown that 8-isoprostane levels are augmented in current smokers compared with NS. ${ }^{27}$

RAGE, a multi ligand receptor for advanced glycation products, has a role in protection against inflammation and tissue injury. ${ }^{28-31}$ We measured RAGE and found significantly higher levels in WPS (cohort II) compared with NS (cohort II).

The anti-inflammatory proteins, such as CC-10 and CC-16 which are secreted by bronchiolar Club cells and are the most abundant proteins in the respiratory tract. They are known to be involved in lung injury and repair processes. ${ }^{32} 33$ We measured CC-10 and CC10/CC-16 in the plasma samples and observed a significant decrease in the CC10/CC-16 levels in CS, WPS and DS compared with NS. CC-10 was significantly lower in CS, but not in WPS or DS. This suggests that DS are at a higher risk of lung injurious responses compared with WPT and CS. Our findings are in agreement with the results of previous studies showing a decrease in CC-10 and CC10/CC-16 levels in smokers compared with NS. 3334 Therefore, based on our observations, CC-10 and CC10/CC-16 may qualify as sensitive biomarkers for tobacco-induced lung injury.

Galectin-3 has been shown to play an important role in the regulation of fibrosis and inflammation. Elevated levels of galectin-3 have been associated with immunological and cardiovascular disorders. ${ }^{35}$ PAI-1 has been implicated in maintaining the haemostasis of the coagulation cascade, and any imbalance in the fibrinolytic system will lead to impaired coagulation that may manifest in cardiovascular diseases. ${ }^{36}$ Both galectin-3 and PAI-1 proteins play a role in orchestrating the normal fibrotic and fibrinolytic systems respectively and elevated levels in the plasma might dysregulate the normal repair and coagulation mechanisms. Our results show that Serpine1/PAI-1 levels in plasma were increasingly higher in CS, WPS and DS compared with NS. Also, the galectin-3 levels in plasma were lower in all smoking groups, although at a non-significant level. Further studies are required to validate these biomarkers in different cohorts with larger sample sizes.

WPS from cohort II, but not cohort I, had elevated levels of urinary en-RAGE, an inflammatory biomarker which plays a role in atherosclerosis and predicts the development of coronary heart disease ${ }^{37}$, and MMP-9, a biomarker for COPD. ${ }^{38}$ This differential result may be explained by the higher frequency and duration of WPT among cohort II smokers. These findings suggest that those who smoke WPT more frequently and over a longer period of time are more likely to develop coronary heart disease and COPD. A longitudinal study using the current cohorts is warranted in order to provide better comparison and validity with the identified mediators.
What this paper adds

What is already known on this subject

- Waterpipe (hookah) tobacco (WPT) is an emerging alternate tobacco product whose use is rapidly increasing, especially in young adults.

- WPT smoking can adversely affect the cardiopulmonary system.

What important gaps in knowledge exist on this topic

- No data are available on the assessment and comparison of systemic biomarkers of inflammation, oxidative stress, lipid mediators and immunity and tissue injury and repair among different smoking groups, including WPT smokers, cigarette smokers and dual WPT and cigarette smokers.

\section{What this paper adds}

- We have identified several biomarkers of exposure in the plasma and urine of WPT smokers, cigarette smokers and dual WPT and cigarette smokers.

- Plasma levels of inflammatory mediators (IL-6, IL-8, IL1 $\beta$ and TNF- $\alpha$ ), and an oxidative stress biomarker (8-isoprostane) in urine were significantly elevated in WPT and cigarette smokers which were further augmented in dual smokers compared with non-smokers.

- A lipid mediator (resolvin E1) showed significant differences in cigarette smokers and dual smokers, compared with nonsmokers. Similarly, the levels of anti-inflammatory mediators CC10/CC-16 were lower in all smoking groups, suggesting that these biomarkers are sensitive for tobacco-induced lung injury.

- These data provide evidence to support policy and public education efforts focused on WPT as well as dual use.

\section{CONCLUSIONS}

Several plasma biomarkers of systemic inflammation, oxidative stress and immunity, and tissue injury and repair were altered in WPT and CS. Further, the concurrent use of WPT and cigarettes appears to be more deleterious than cigarette or WPT smoking alone, aggravating pulmonary and cardiovascular conditions. Our study offers evidence to counter the public perception that WPT smoking is safer than cigarette smoking, ${ }^{1-3}$ and to inform WPT control policy efforts.

Contributors NAK, NOFK, IR conceived and designed the experiments, wrote and edited the manuscript. KPS did data analysis, wrote and edited the manuscript. IR and NOFK obtained research funding and study design and experimental plans/ assays. NAK, GL, NOK, FKP recruited the volunteers. NAK, GL, QW, DY, SRM, NOK, FKP performed the experiments. All authors contributed to manuscript preparation and approved the final version prior to submission.

Funding This work was supported in part by a National Institutes of Health (NIH) Grants, NIH 1R01HL135613, NIH 1R01HL085613 and the Food and Drug Administration (FDA) Center for Tobacco Products (CTP) via the National Institute on Drug Abuse (NIDA) of the NIH, NIHFDACTP grant\# 1R01DA042470 (to Irfan Rahman) and R01DA042471 (to Nada O.F. Kassem). The content is solely the responsibility of the authors and does not necessarily represent the official views of the $\mathrm{NIH}$ or the FDA.

Competing interests None declared.

Patient consent for publication Not required.

Ethics approval Cohort I: approved by the General Clinical Research Center of the University of Rochester Research Subjects Review Board (IRB\#RSRB00063526). Cohort II: Study procedures were approved by the San Diego State University Institutional Review Board (IRB\# 2445100).

Provenance and peer review Not commissioned; externally peer reviewed. 
Data availability statement All data relevant to the study are included in the article or uploaded as supplementary information.

ORCID iD

Irfan Rahman http://orcid.org/0000-0003-2274-2454

\section{REFERENCES}

1 Maziak W, Taleb ZB, Bahelah R, et al. The global epidemiology of waterpipe smoking. Tob Control 2015;24 Suppl 1:i3-12.

2 El-Zaatari ZM, Chami HA, Zaatari GS. Health effects associated with waterpipe smoking. Tob Control 2015;24(Suppl 1):i31-43.

3 Waziry R, Jawad M, Ballout RA, et al. The effects of waterpipe tobacco smoking on health outcomes: an updated systematic review and meta-analysis. Int J Epidemiol 2017:46:32-43.

4 Warnakulasuriya S. Waterpipe smoking, oral cancer and other oral health effects. Evid Based Dent 2011;12:44-5.

5 Daher N, Saleh R, Jaroudi E, et al. Comparison of carcinogen, carbon monoxide, and ultrafine particle emissions from narghile waterpipe and cigarette smoking: sidestream smoke measurements and assessment of second-hand smoke emission factors. Atmos Environ 2010;44:8-14.

6 Hammal F, Chappell A, Wild TC, et al. 'Herbal' but potentially hazardous: an analysis of the constituents and smoke emissions of tobacco-free waterpipe products and the air quality in the cafés where they are served. Tob Control 2015;24:290-7.

7 Javed F, Al-Kheraif AA, Rahman I, et al. Comparison of clinical and radiographic periodontal status between habitual Water-Pipe smokers and cigarette smokers. J Periodontol 2016;87:142-7.

8 Bhatnagar A, Maziak W, Eissenberg T, et al. Water pipe (Hookah) smoking and cardiovascular disease risk: a scientific statement from the American Heart Association. Circulation 2019:139:e917.

9 Bahtouee M, Maleki N, Nekouee F. The prevalence of chronic obstructive pulmonary disease in hookah smokers. Chron Respir Dis 2018:15:165-72.

10 Raad D, Gaddam S, Schunemann HJ, et al. Effects of water-pipe smoking on lung function: a systematic review and meta-analysis. Chest 2011;139:764-74.

11 Chaouachi KT. The narghile (hookah, shisha, goza) epidemic and the need for clearing up confusion and solving problems related with model building of social situations. ScientificWorldJournal 2007;7:1691-6.

12 Hessami Z, Masjedi MR, Mortaz E, et al. Evaluation of dual tobacco smoking (water pipe and cigarettes) and associated factors in adults in Tehran. Tanaffos 2016;15:180-6.

13 Deveci SE, Deveci F, Acik Y, et al. The measurement of exhaled carbon monoxide in healthy smokers and non-smokers. Respir Med 2004;98:551-6.

14 Kassem NOF, Kassem NO, Liles S, et al. Waterpipe device cleaning practices and disposal of waste associated with waterpipe tobacco smoking in homes in the USA. Tob Control 2020;29(Supp 2):s123-30.

15 Kassem NOF, Kassem NO, Jackson SR, et al. Benzene uptake in Hookah smokers and non-smokers attending Hookah social events: regulatory implications. Cancer Epidemiol Biomarkers Prev 2014;23:2793-809.

16 Pan Z, Yu H, Liao J-L. Probing cellular and molecular mechanisms of cigarette smoke-induced immune response in the progression of chronic obstructive pulmonary disease using multiscale network modeling. PLoS One 2016:11:e0163192.

17 Pinto-Plata V, Toso J, Lee K, et al. Profiling serum biomarkers in patients with COPD: associations with clinical parameters. Thorax 2007:62:595-601.
18 Pinto-Plata VM, Müllerova $\mathrm{H}$, Toso JF, et al. C-reactive protein in patients with COPD, control smokers and non-smokers. Thorax 2006:61:23-8.

19 Wedzicha JA, Seemungal TA, MacCallum PK, et al. Acute exacerbations of chronic obstructive pulmonary disease are accompanied by elevations of plasma fibrinogen and serum IL-6 levels. Thromb Haemost 2000;84:210-5.

20 Karadag F, Karul AB, Cildag 0, et al. Biomarkers of systemic inflammation in stable and exacerbation phases of COPD. Lung 2008;186:403-9

21 Garcia-Rio F, Miravitlles M, Soriano JB, et al. Systemic inflammation in chronic obstructive pulmonary disease: a population-based study. Respir Res 2010;11:63.

22 Khan NA, Sundar IK, Rahman I. Strain- and sex-dependent pulmonary toxicity of waterpipe smoke in mouse. Physiol Rep 2018;6:e13579.

23 Sundar IK, Yao H, Rahman I. Oxidative stress and chromatin remodeling in chronic obstructive pulmonary disease and smoking-related diseases. Antioxid Redox Signal 2013:18:1956-71.

24 Jukema JB, Bagnasco DE, Jukema RA. Waterpipe smoking: not necessarily less hazardous than cigarette smoking : Possible consequences for (cardiovascular) disease. Neth Heart J 2014;22:91-9.

25 Javed F, ALHarthi SS, BinShabaib MS, et al. Toxicological impact of waterpipe smoking and flavorings in the oral cavity and respiratory system. Inhal Toxicol 2017:29:389-96.

26 Mokeem SA, Alasqah MN, Michelogiannakis D, et al. Clinical and radiographic periodontal status and whole salivary cotinine, IL-1 $\beta$ and IL-6 levels in cigarette- and waterpipe-smokers and E-cig users. Environ Toxicol Pharmacol 2018;61:38-43.

27 van der Plas A, Pouly S, de La Bourdonnaye G, et al. Influence of smoking on levels of urinary 8-iso prostaglandin F2 $\alpha$. Toxicol Rep 2019;6:18-25.

28 Sparvero LJ, Asafu-Adjei D, Kang R, et al. RAGE (Receptor for Advanced Glycation Endproducts), RAGE ligands, and their role in cancer and inflammation. J Trans/ Med 2009;7:17.

29 Alexiou P, Chatzopoulou M, Pegklidou K, et al. RAGE: a multi-ligand receptor unveiling novel insights in health and disease. Curr Med Chem 2010;17:2232-52.

30 Yan SF, Ramasamy R, Schmidt AM. Soluble RAGE: therapy and biomarker in unraveling the RAGE axis in chronic disease and aging. Biochem Pharmacol 2010;79:1379-86.

31 Maillard-Lefebvre H, Boulanger E, Daroux M, et al. Soluble receptor for advanced glycation end products: a new biomarker in diagnosis and prognosis of chronic inflammatory diseases. Rheumatology 2009;48:1190-6.

32 Singh G, Katyal SL. Clara cells and Clara cell 10 kD protein (CC10). Am J Respir Cell Mol Biol 1997:17:141-3.

33 Broeckaert F, Clippe A, Knoops B, et al. Clara cell secretory protein (CC16): features as a peripheral lung biomarker. Ann N Y Acad Sci 2000;923:68-77.

34 Chen J, Lam S, Pilon A, et al. The association between the anti-inflammatory protein CC10 and smoking status among participants in a chemoprevention trial. Cancer Epidemiol Biomarkers Prev 2007;16:577-83.

35 de Boer RA, Voors AA, Muntendam P, et al. Galectin-3: a novel mediator of heart failure development and progression. Eur I Heart Fail 2009;11:811-7.

36 Essa ES, El Wahsh RA. Association between plasminogen activator Inhibitor-1-675 4G/5G insertion/deletion polymorphism and chronic obstructive pulmonary disease. COPD 2016;13:756-9.

37 Ligthart S, Sedaghat S, Ikram MA, et al. EN-RAGE: a novel inflammatory marker for incident coronary heart disease. Arterioscler Thromb Vasc Biol 2014;34:2695-9.

38 Abd El-Fatah MF, Ghazy MA, Mostafa MS, et al. Identification of MMP-9 as a biomarker for detecting progression of chronic obstructive pulmonary disease. Biochem. Cell Biol. 2015:93:541-7. 